\title{
Development and Evaluation of Internal and External Predictability of Metoclopramide Hydrochloride Modified Release Formulations: An Establishment of Level A In vitro and In vivo Correlation
}

\author{
Ramesh Narayanasamy ${ }^{1 *}$, Ramakrishna Shabaraya ${ }^{1}$ \\ ${ }^{1}$ Department of Pharmaceutics, Srinivas College of Pharmacy, Farengipete Post, Mangalore- 574143, Karnataka, INDIA.
}

\begin{abstract}
The objective of this study was to develop an in vitro-in vivo correlation (IVIVC) model for hydrophilic matrix sustained-release (SR) Metoclopramide formulations. The in vitro release characteristics of the drug were determined using USP apparatus II at $50 \mathrm{rpm}, \mathrm{pH}$ 6.8. In vivo plasma concentrations and pharmacokinetic parameters in healthy human subjects were obtained after administering oral dose, developed SR formulations and marketed immediate-release (IR) products. The similarity factor $f 2$ was used to compare the dissolution data. The IVIVC model was developed using pooled fraction dissolved and fraction absorbed of developed SR formulations i.e. fast, medium and slow release and marketed immediate-release (IR) products. An in vitro-in vivo correlation (IVIVC) was established for sustained release tablet by deconvolution using data from an immediate-release treatment as the characteristic response. To assess the correlation between in vitro dissolution uniqueness and in vivo absorption performance of Metoclopramide sustained release (SR) and immediate release (IR) tablet in human subjects. The established IVIVC was evaluated internally by predicting data used to develop and externally by predicting data not originally included in developing the IVIVC model. The observed low prediction errors for Cmax and AUC demonstrated that the Metoclopramide IVIVC model was valid.
\end{abstract}

Keywords: Metoclopramide Hydrochloride, Modified Release, Level A Correlation, Internal And External Prediction Errors.

\section{INTRODUCTION}

In vitro in vivo correlation is a predictive mathematical model describing the relationship between the in vitro property of dosage form and in vivo response. In vitro property is a rate of drug release and in vivo response is the amount of drug absorbed. In vitro in vivo correlation plays a vital role in the early stage of product development, substitute for in vivo bioavailability and to support biowaivers, to facilitate scale-up and post approval changes, and to set significant dissolution specifications. In vitro in vivo correlation is established to facilitate dissolution test to be used as surrogacy for bio study and it benefit the pharmaceutical manufacturer in terms of time and cost on bioequivalence study. $^{7-10}$

In vitro in vivo correlation models will be useful for optimizing the SR (sustained release) dosage forms, otherwise predict in vivo performance of the SR dosage forms based on in vitro dissolution data. The FDA guidance has identified three In vitro in vivo correlation models: namely, level A, B, and C models. ${ }^{6}$ Numerous investigations have been undertaken to develop In vitro in vivo correlation models, a level A correlation utilize the complete time course of in vitro dissolution and in vivo input and recognized model of choice for achieve biowaivers or
Submission Date: 01-02-2017; Revision Date: 03-03-2017; Accepted Date: 18-03-2017

DOI: 10.5530/ijper.51.2s.45 Correspondence: Ramesh N, Department of Pharmaceutics Srinivas College of Pharmacy, Farengipete Post, Mangalore, Karnataka - 574143, INDIA Ph: + 918242232700 Fax: + 918242274725 E mail: ramesh7779@gmail. com 
setting of dissolution specifications. ${ }^{7-16}$ However, level $\mathrm{B}$ and $\mathrm{C}$ models may be used in the initial stages of formulation development to inspect level A In vitro in vivo correlation models shall be used. ${ }^{6}$

The present work aim was to develop and establish the internal and external predictability of level A In vitro in vivo correlation models for the three Metoclopramide sustained release and one immediate release formulation were evaluated. The rationale behind the study was develop Metoclopramide In vitro in vivo correlation models, so that it can serve as a surrogate for in vivo bioavailability and to support biowaivers and which in turn reduce the cost of the optimization process.

\section{MATERIALS AND METHODS}

\section{Chemicals and Reagents}

Metoclopramide Hydrochloride \& Cisapride were supplied by Adcock Ingram Healthcare Pvt. Ltd. (Bangalore, India). The HPMCK100M supplied by Colorcon Asia Pvt Ltd. (Goa, India). Avicel, Magnesium stearate and talc were procured from local supplier.

\section{Formulation of Tablets}

Sustained release formulations, each tablet containing $10 \mathrm{mg}$ of Metoclopramide hydrochloride, prepared by weighing the amount of active ingredients, polymers (HPMC), avicel magnesium stearate and talc in different ratio and blended to get homogeneous mixture. Tablets were prepared by direct compression on a single punch machine (10 $\mathrm{mm}$ bi-flat round shaped punches). The Metoclopramide hydrochloride formulations were prepared at three different release rates i.e. fast, medium and slow sustained release tablets. ${ }^{1-3}$

\section{In vitro release studies}

The dissolution performance of Metoclopramide hydrochloride determined by using a USP dissolution apparatus 2 (paddle method). The release studies were performed in $\mathrm{pH} 1.2,4.5,5.5,6.8,7.4(900 \mathrm{ml})$ at 37.0 $\pm 0.5{ }^{\circ} \mathrm{C}$ and a rotation speed of paddle was $50 \& 75$ $\mathrm{rpm}$. Samples $(5 \mathrm{ml})$ were collected at $0.0,0.5,1.0,1.5$, 2.0, 3.0, 4.0, 6.0, 8.0, 12.0, 18.0 and 24.0 hours. and replaced with fresh medium at various interval times. The amount of released Metoclopramide hydrochloride was analyzed by using UV-Visible spectrophotometric at a wavelength of $309 \mathrm{~nm}$.

\section{Clinical pharmacokinetic study}

This was an open-label, single dose, four-treatment crossover study by using six healthy volunteers under fasting condition. Subjects were given informed consent prior to participation and study was approved by the ethics committee. Subjects randomly allocated treatment as per the randomization schedule, such a way all subjects would receive four formulations upon completion of the study. Blood samples were collected at predetermined time $0.0,0.33,0.67,1.0,1.33,1.67,2.0,3.0,4.0$, 6.0, 8.0, 10.0, 12.0, 16.0, 18.0 and 24.00 hours post-dose. Washout period of at least 07 days between dosage. ${ }^{4}$ Plasma samples were stored in an upright position below $-20^{\circ} \mathrm{C}$ till completion of analysis. Plasma samples were estimated for Metoclopramide by using a validated LCMS/MS with liquid liquid extraction method. Chromatographic separation achieved on a Eclipse XDB C18 (100 mm x $4.6 \mathrm{~mm}, 3.5 \mu \mathrm{m})$ column using a mobile phase consisting of methanol and ammonium acetate buffer $5 \mathrm{mM}(50: 50 \mathrm{v} / \mathrm{v})$. The RT of Metoclopramide hydrochloride and internal standard was 1.1 and 2.1 minutes. The method was validated over a concentration range of $0.532 \mathrm{ng} / \mathrm{mL}$ to $201.005 \mathrm{ng} / \mathrm{mL}$ for Metoclopramide. The lower limit of quantization (LLLQ) was established at $0.532 \mathrm{ng} / \mathrm{mL}$ for Metoclopramide. Plasma sample analysis were performed by using a validated high performance liquid chromatography mass spectrometric method. ${ }^{5}$

\section{Dissolution data analysis}

The in vitro drug release was calculated by using similarity factor $(2)$ and dissolution profile of all formulations were determined by scheming the cumulative percent of Metoclopramide hydrochloride versus time. The in-vitro drug release profile of the three SR formulations were compared using the similarity factor $(f 2)$ as mentioned in the following equation:

$\mathrm{f} 2=50 \log \left[\begin{array}{c}\mathrm{n} \\ 1+1 / \mathrm{n} \sum\left(\mathrm{Rt}-\mathrm{T}_{\mathrm{t}}\right)^{2} \\ \mathrm{t}=1\end{array}\right] \times 100 \quad$ (Equation 1) where $R_{t}$ and $T_{t}$ are the percent dissolved at each time point for the reference and test product respectively.

The $f_{2}$ equation is a logarithmic transformation of the sum of squares of the difference between test and reference formulation. The $f_{2}$ average difference between 0 and 100. If the value of $f_{2}$ is less than 50 dissolution profiles wee considered dissimilar. If $f_{2}$ value 50 suggests that the reference and test product release curves differ by at least $10 \%$ and $f_{2}$ values greater than 50 (between 50 and 100) make sure similarity or uniformity between two dissolution profiles. The percent drug dissolved versus time calculated by using weibull method.

\section{Clinical pharmacokinetic data analysis}

The plasma concentration-time data were estimated by using Phoenix 6.4.0 version software. Maximum plasma 
concentration observed for the subject was Cmax and time to take place was Tmax. Area under the plasma concentration-time profile estimated by the trapezoidal method. The rate constant $(\mathrm{K})$ describing the terminal slope of mean plasma concentration- time profile was determined by linear least squares fitting of the natural logarithm (Ln) transformed terminal plasma concentrations vs. time to the equation for a straight line. The elimination rate constant was estimated by linear regression of linear portion of logarithm of the concentrations versus time. The percent of drug absorbed versus time estimated by using deconvolution technique.

\section{In vitro In vivo Correlation}

Level A correlation was developed as mentioned in the FDA and USP guidelines by using the data obtained in the in vivo study. The percent of drug dissolved was estimated by plotting time points against the drug release profile and the fraction of drug absorbed was derived by using Wagner-Nelson method. Linear regression analysis was used to inspect the relationship between percent of drug dissolved and percent of drug absorbed. The correlation developed by using in vitro and in vivo data.

\section{IVIVC Model validation}

The objective is to validate the prediction of in vivo performance from in vitro data. The validation explain how well the developed IVIVC model predicts the in vivo data when in vitro data applied. The percentage prediction error of Cmax and AUC estimated by using equation mentioned below:

$$
\% \mathrm{PE}_{\mathrm{C} \max }=\frac{\text { observed }- \text { predicted }}{\text { observed }} \times 100
$$

Internal validation includes the cross validation of one formulation with other three formulation used for IVIVC model development. Internal validation is optional for IVIVC analysis and provides base for the suitability of the model. and external validation is based on how good IVIVC predicts the additional set of data. As described on FDA guidance IVIVC model is valid when the internal predictability (absolute PE) of Cmax and AUC for each formulation should not exceed 15\% and with the average should not exceed 10\% (percentage), and for external validation the prediction errors of Cmax and AUC should not exceed $10 \%$ and values between $10 \%$ to $20 \%$ and more than $20 \%$ is not acceptable.

\section{RESULTS}

\section{In vitro studies}

The in vitro drug release studies were conducted at different $\mathrm{pH}$ conditions (namely $\mathrm{pH} 1.2,4.5,5.5,6.8,7.4$ ) to check the release and to select the optimum $\mathrm{pH}$ condition for the drug. The percentage fraction dissolved for Metoclopramide hydrochloride of marketed immediate release, fast, medium and slow sustained release tablets and percentage drug release at different time points are presented in Table 1 \& Figure 1.

\section{DISCUSSION}

In vitro and in vivo studies were performed for the developed sustained release formulation i.e. fast, medium and slow sustained release tablets. Dissolution studies were performed at $\mathrm{pH} 1.2$ for $50 \mathrm{rpm}$, the release of the drug was unfinished and showed maximum up to $31.98 \%$, $\mathrm{pH} 4.5$ at $50 \mathrm{rpm}$, the drug release was slow over the last time point and maximum release up to $85.64 \%$ $\mathrm{pH} 5.5$ at $50 \mathrm{rpm}$ drug release was homogeneous and observed $94.50-99.23 \%$ over 24 hours, $\mathrm{pH} 6.8$ and $\mathrm{pH}$ 7.4 at $50 \mathrm{rpm}$, drug released up to $91.08-95.21 \%$ and $89.57-90.47 \%$. To check rpm effect dissolution tests performed at $\mathrm{pH} 1.2, \mathrm{pH} 4.5, \mathrm{pH} 5.5, \mathrm{pH} 6.8$ and $\mathrm{pH}$ 7.4 at $75 \mathrm{rpm}$ not much variations observed. Therefore for $\mathrm{pH} 5.5$ at $50 \mathrm{rpm}$ formulation optimized as slow, medium and fast release. The in vitro release characteristics Metoclopramide hydrochloride Sustained Release formulations were determined and the percentage drug release at various time intervals. The optimized formulation at $\mathrm{pH} 5.5$ were reformulated i.e. Metoclopramide hydrochloride SR Tablets (Slow, Medium \& Fast) and dissolution test performed for test and reference formulation at pH 6.8 as per the USP method. The percentage fraction dissolved for Metoclopramide hydrochloride are in the rank order of marketed immediate release, fast, medium and slow sustained release tablets and percentage drug release at different time points are presented in Table $1 \&$ Figure 1 . The $\mathrm{f} 2$ values are calculated for fast versus slow $\mathrm{f} 2$ value is 47.12 at the borderline, fast versus medium and medium versus slow formulation observed higher than 50 confirms and for formulation that these dissolution mediums are indistinguishable and ensures sameness or equivalence between the two dissolution profiles.

The mean Metoclopramide pharmacokinetic parameters AUClast and AUCINF_obs were reasonably higher for marketed release tablet compare to the test formulation. Followed by lower elimination rate and higher half life for fast and medium release observed compare to slow release and marketed tablet. The mean pharmacokinetic results are presented in Figure 2 and Table 2.

A Level A correlation was developed by building IVIVC plot using percent dissolved versus percent absorbed for all three formulations. The slope of the best-fit line was 
observed for percentage of drug dissolved and the percentage of drug absorbed. The correlation coefficient $\left(\mathrm{r}^{2}\right)$ for was $0.9025,0.8905$ and 0.8685 are presented in Figure 3, 4 and 5 respectively. A good linear regression relationship was observed.

\section{IVIVC Validation}

The percentage prediction errors for $\mathrm{C}_{\max }$ and $\mathrm{AUC}$ were calculated by using marketed Metoclopramide hydrochloride immediate release tablets as a target formulation. The $\mathrm{C}_{\max }$ prediction errors for the fast, medium and slow

\section{Table 1: Percentage Cumulative Release of Metoclopramide Hydrochloride SR Tablets (E2 Slow, E4 Medium \&}

\begin{tabular}{|c|c|c|c|c|c|c|}
\hline \multirow[b]{2}{*}{ Time } & \multicolumn{2}{|c|}{ Slow (E2) } & \multicolumn{2}{|c|}{ Medium (E4) } & \multicolumn{2}{|c|}{ Fast (E5) } \\
\hline & $\%$ Release & $\begin{array}{c}\% \text { Cumulative } \\
\text { Release }\end{array}$ & $\%$ Release & $\%$ Cumulative Release & $\%$ Release & $\begin{array}{c}\% \text { Cumulative } \\
\text { Release }\end{array}$ \\
\hline 0.0 & 0.00 & 0.0 & 0.00 & 0.00 & 0.00 & 0.00 \\
\hline 0.50 & 12.60 & 10.70 & 15.40 & 13.00 & 19.20 & 11.90 \\
\hline 1.00 & 17.70 & 17.77 & 20.50 & 20.59 & 24.20 & 24.31 \\
\hline 1.50 & 26.10 & 26.20 & 31.70 & 31.81 & 34.30 & 34.43 \\
\hline 2.00 & 32.90 & 33.05 & 38.50 & 38.68 & 42.60 & 42.79 \\
\hline 2.50 & 39.60 & 39.78 & 49.70 & 49.91 & 53.50 & 53.74 \\
\hline 3.00 & 48.00 & 48.22 & 57.40 & 57.68 & 61.00 & 61.30 \\
\hline 4.00 & 53.90 & 54.17 & 63.40 & 63.72 & 67.70 & 68.04 \\
\hline 6.00 & 58.10 & 58.40 & 69.30 & 69.65 & 76.10 & 76.48 \\
\hline 8.00 & 66.50 & 66.82 & 76.20 & 76.59 & 79.40 & 79.82 \\
\hline 10.00 & 73.30 & 73.67 & 80.50 & 80.92 & 85.20 & 85.64 \\
\hline 12.00 & 78.30 & 78.71 & 86.50 & 86.95 & 89.40 & 89.87 \\
\hline 18.00 & 85.10 & 85.54 & 92.50 & 92.98 & 95.30 & 95.80 \\
\hline 24.00 & 91.80 & 92.27 & 95.00 & 95.51 & 98.60 & 99.13 \\
\hline
\end{tabular}

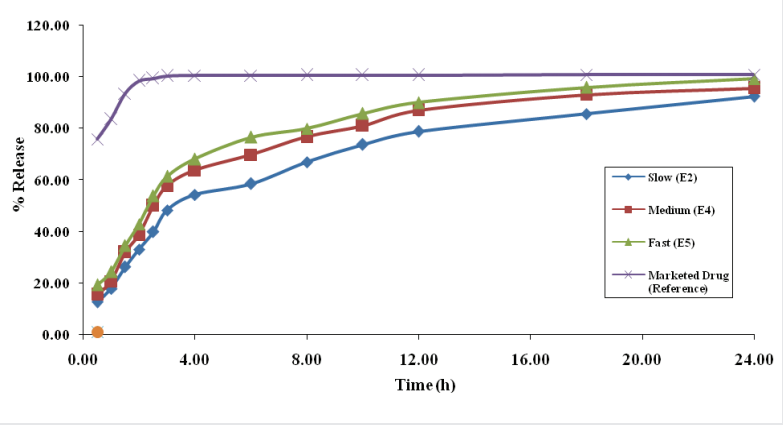

Figure 1: Percentage Cumulative Release of Metoclopramide Hydrochloride SR Tablets (Slow, Medium, Fast \& Marketed Drug)

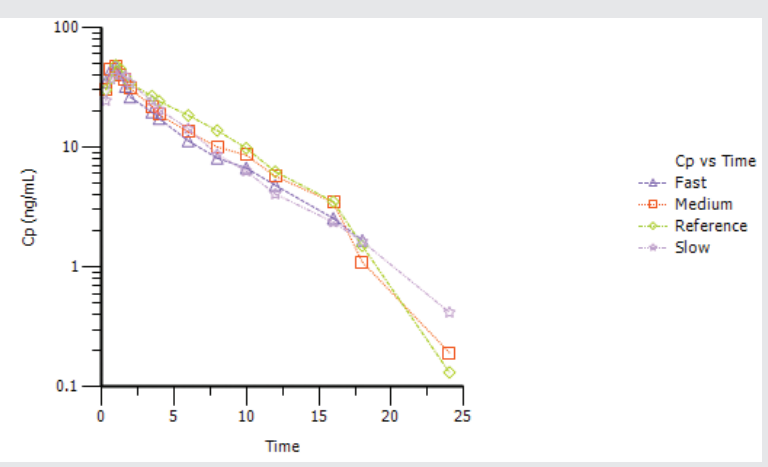

Figure 2: Mean plasma concentrations versus time of Metoclopramide Hydrochloride for Marketed Immediate Release and Sustained Release Tablet (Fast, Medium \& Slow)

$\begin{gathered}\text { Table 2: Mean Pharmacokinetic Parameter of Metoclopramide Hydrochloride for Marketed Immediate } \\
\text { Release and Sustained Release Tablet (Fast, Medium \& Slow) }\end{gathered}$
\begin{tabular}{|c|c|c|c|c|c|c|} 
Formulation & Kel (1/hr) & Half life (hr) & Tmax (hr) & $\begin{array}{c}\text { Cmax } \\
\text { (ug/mL) }\end{array}$ & $\begin{array}{c}\text { AUClast } \\
\text { (hr*ug/mL) }\end{array}$ & $\begin{array}{c}\text { AUCINF_obs } \\
\text { (hr*ug/mL) }\end{array}$ \\
\hline Immediate Release Tablet & 0.195 & 3.587 & 1.11 & 49.06 & 267.393 & 273.694 \\
\hline Fast Sustained Release Tablet & 0.186 & 3.749 & 0.89 & 48.433 & 205.467 & 214.506 \\
\hline Medium Sustained Release Tablet & 0.199 & 3.729 & 0.89 & 48.004 & 230.418 & 238.671 \\
\hline Slow Sustained Release Tablet & 0.221 & 3.564 & 1.00 & 48.366 & 222.996 & 230.834 \\
\hline
\end{tabular}




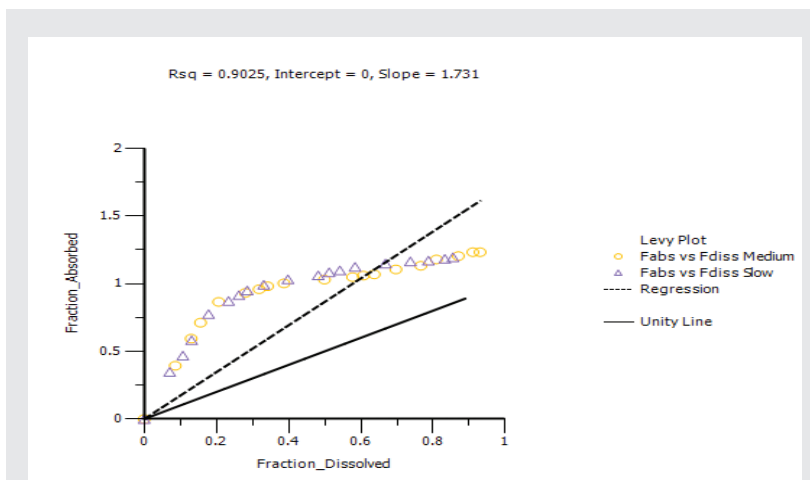

Figure 3: IVIVC Model Linear Regression Percentage Dissolved and Percentage Absorbed for Metoclopramide Hydrochloride and Slow SR Tablet

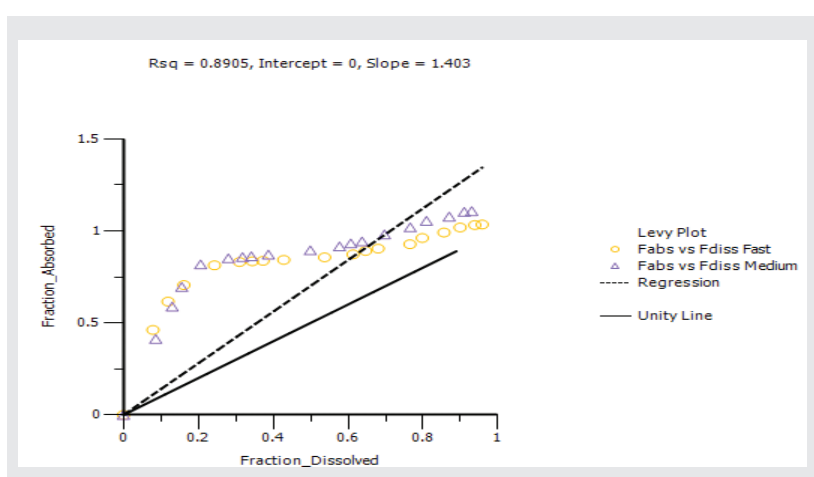

Figure 4: IVIVC Model Linear Regression Percentage Dissolved and Percentage Absorbed for Metoclopramide Hydrochloride Fast and Medium SR Tablet

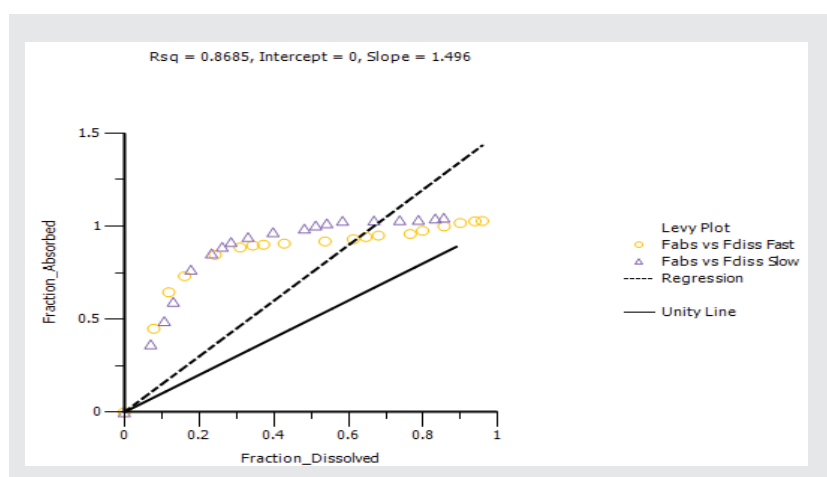

Figure 5: IVIVC Model Linear Regression Percentage Dissolved and Percentage Absorbed for Metoclopramide Hydrochloride Fast and Slow SR Tablet

formulations were found to be $-12.356 \%,-16.049 \%$ and $-24.612 \%$ respectively and AUC values were $-22.484 \%$, $7.461 \%$ and $7.349 \%$ for respectively refer Table 3.

The $\mathrm{C}_{\max }$ prediction errors prediction errors for Metoclopramide hydrochloride SR tablets i.e. fast, medium, slow and marketed immediate release formulations were estimated and results are observed -56.178, -58.401, -62.407 and 3.349, respectively and for AUC values are $-38.758,-40.158,-41.836$ and -27.405 respectively. The
Table 3: Prediction errors (\%) associated with Cmax and AUC for Metoclopramide Hydrochloride Fast, Medium and Slow SR Tablets

\begin{tabular}{|c|c|c|}
\hline Formulation & Parameter & \%PE \\
\hline Fast Internal & AUClast & -22.484 \\
\hline Fast Internal & Cmax & -12.356 \\
\hline Medium Internal & AUClast & 7.461 \\
\hline Medium Internal & Cmax & -16.049 \\
\hline Slow Internal & AUClast & 7.349 \\
\hline Slow Internal & Cmax & -24.612 \\
\hline
\end{tabular}

study was conducted to examine, Level A IVIVC relating the complete time-course of plasma concentrations was developed and validated internally and externally for Metoclopramide hydrochloride formulation i.e. fast, medium and slow SR tablets and marketed immediate release tablet. The validation of internal and external predictability was successfully accomplished. The prediction error for $\mathrm{C}_{\max }$ and $\mathrm{AUC}$ was within the specified limit as per the FDA guidance hence, the IVIVC is well thought-out as validated both in terms of internal and external validation. Thus, this IVIVC model may be used during process change, site change, to reduce the number of human studies during the formulation development and serve as a surrogate for in vivo bioavailability, to support biowaivers, to support dissolution methods and specification settings. It can also assist in quality control for during the scale-up and postapproval changes (SUPAC).

\section{CONCLUSION}

The developed in vitro methods can act as a surrogate for in vivo bioavailability study and support biowaivers, assist in quality control during scale-up and post-approval changes. It may be useful in predicting the variation in site change, process changes and to predict the absorption performance of Metoclopramide Hydrochloride products with different release rates.

\section{ACKNOWLEDGEMENT}

The authors would like to thank Srinivas College of Pharmacy, Sequent Research Limited \& Adcock Ingram Healthcare Pvt. Ltd. (Bangalore, India), Colorcon Asia Pvt Limited, India and Phoenix Winnonlin Certara software Hyderabad, India for granting support to carry out the work for providing required facilities to carry out this research work. 


\section{CONFLICT OF INTEREST}

The authors confirm that this article content has no conflict of interest.

\section{ABBREVIATION USED}

nm: Nanometer, Liquid chromatography with tandem mass spectrometry (LC-MS-MS); ${ }^{\circ} \mathbf{C}$ : Degree Celsius; ml: Milliliter; ng/mL: Nanogram/milliliter; HPMC: Hydroxypropyl methylcellulose; mm: Millimeter; USP: United State of Pharmacopeia: FDA: Food and Drug Administration; Cmax: Maximum plasma concentration; Tmax: Time of the maximum plasma concentration; AUClast: Area under the plasma concentration-time curve from time zero to the time of the last quantifiable concentration; AUC INF_obs: Area under the plasma concentration-time curve from time zero extrapolated to the infinite time; Kel: Elimination rate constant; \%: Percentage.

\section{REFERENCES}

1. Hamed E, Sakr A. Application of multiple response optimization technique to extended release formulations design. J. Control Release. 2001;73(2):329-38. https://doi.org/10.1016/S0168-3659(01)00356-X.

2. Sayed IAR, Gamal MM, Mahmoud BE. Preparation and comparative evaluation of sustained release Metoclopramide hydrochloride matrix tablets. Saudi Pharmaceutical Journal. 2009;S17(4):283-8.

3. Hasan El, Amro BI, Arafat T, Badwan AA. Assessment of controlled release of hydrophilic matrix formulation for Metoclopramide Hydrochloric acid. Eur. J. Pharm. Biopharm. 2003;S55(3):339-44. https://doi.org/10.1016/S09396411(03)00022-5.

4. Yan M, Li HD, Chen BM, Liu XL, Zhu YG. Determination of metoclopramide in human plasma by LC-ESI-MS and its application to bioequivalance studies. J Chromatogr B AnalytTechnol Biomed Life Sci. 2010;878(11-12):883-7. https://doi.org/10.1016/j.jchromb.2010.02.006; PMid:20189472.

5. Jaswanth KI, Rajasekhar D, Ramesh M, Venkateswarlu P. Sensitive and selective liquid chromatography-tandem mass spectrometry method for the determination of Metoclopramide in human plasma: application to a bioequivalence study. Biomed. Chromatogr. 2010;24:1006-14.

6. Food and Drug Administration. 1997. Guidance for industry, extended release oral dosage forms: Development, evaluation, and application of in vitro/ in vivo correlations.

7. Takka S, Sakr A, Goldberg A. Development and Validation of an In vitroIn vivo Correlation for Buspirone Hydrochloride Extended Release Tablets. Journal of Controlled Release.2003;88(1):147-57. https://doi.org/10.1016/ S0168-3659(02)00490-X.

8. Eddington ND, Ashraf M, Augsburger LL. Identification of formulation and manufacturing variables that influence in vitro dissolution and in vivo bioavailability of propranolol hydrochloride tablets. Pharm Dev Technol 1998;3(4):535-47. https://doi.org/10.3109/10837459809028636; PMid:9834957

9. Hayes S, Dunne A, Smart T. Interpretation and optimization of the dissolution specifications for a modified release product with an in vivo-in vitro correlation (IVIVC). J Pharm Sci 2004;93(3):571-81. https://doi.org/10.1002/jps.10552; PMid:14762896

10. Emami J. In vitro-in vivo correlation: from theory to applications. J Pharm Pharm Sci 2006;9(2):169-89. PMid:16959187.

11. Mahayni H, Rekhi GS, Uppoor RS. Evaluation of "external" predictability of an in vitro-in vivo correlation for an extended-release formulation containing metoprolol tartrate. J Pharm Sci 2000;89(10):1354-61. https://doi. org/10.1002/1520-6017(200010)89:10<1354::AID-JPS13>3.0.CO;2-P.

12. Mandal U, Ray KK, Gowda V. In-vitro and in-vivo correlation for two gliclazide extended-release tablets. J Pharm Pharmacol 2007;59(7):971-6. https://doi. org/10.1211/jpp.59.7.0009; PMid:17637192.

13. Sirisuth N, Eddington ND. The influence of first pass metabolism on the development and validation of an IVIVC for metoprolol extended release tablets. Eur J Pharm Biopharm 2002;53(3):301-9. https://doi.org/10.1016/ S0939-6411(01)00248-X.

14. Huang YB, Tsai YH, Yang WC, et al. Once-daily propranolol extended-release tablet dosage form: formulation design and in vitro/in vivo investigation. Eur J Pharm Biopharm. 2004;58(3):607-14. https://doi.org/10.1016/j. ejpb.2004.03.037; PMid:15451535.

15. Dutta S, Qiu Y, Samara E, et al. Once-a-day extended-release dosage form of divalproex sodium III: development and validation of a Level A in vitroin vivo correlation (IVIVC). J Pharm Sci 2005;94(9):1949-56. https://doi. org/10.1002/jps.20387; PMid:16052544.

16. 16. Jolly MS, Mayur GS, Rajashree CM. Drug release and swelling kinetics of directly compressed glipizide sustained-release matrices: Establishment of level A IVIVC. Journal of Controlled Release. 2008;129(1):49-58. https:// doi.org/10.1016/j.jconrel.2008.03.016; PMid:18456362

\section{SUMMARY}

- Level A in vitro and in vivo correlation was developed by comparing percent dissolved versus the percent absorbed of Metoclopramide Hydrochloride fast, medium and slow sustained release and marketed immediate release tablets.

- In vitro and in vivo correlation can be used in the development of new drug to decrease the number of human studies during the formulation development and optimization. 


\section{About Authors}

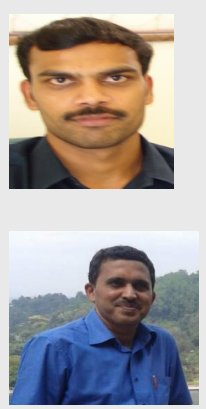

Ramesh. N: Research Scholar in Department of Pharmaceutics, Srinivas College of Pharmacy, Mangalore, India.

Dr. A. R. Shabaraya: Principal and Director, Srinivas College of Pharmacy, Mangalore, India.

Cite this article: Ramesh N, Shabaraya R. Development and Evaluation of Internal and External Predictability of Metoclopramide Hydrochloride Modified Release Formulations: An Establishment of Level A In vitro and In vivo Correlation. Indian J of Pharmaceutical Education and Research. 2017;51(2S):S17-S23. 